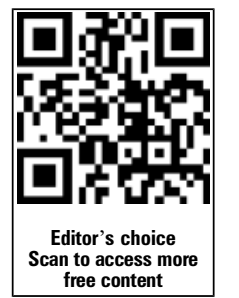

'Department of Medicine, Shrewsbury and Telford Hospital NHS Trust, Princess Royal Hospital, Telford, Shropshire, UK ${ }^{2}$ Department of Palliative Medicine, Douglas Macmillan Hospice, Stoke-on-Trent, Staffordshire, UK

${ }^{3}$ Department of Anaesthetics, University Hospital of North Staffordshire NHS Trust, Stoke-on-Trent, Staffordshire, UK

\section{Correspondence to}

Dr Matthew James Billingham, Department of Medicine, Shrewsbury and Telford Hospital NHS Trust, Princess Royal Hospital, Apley Castle, Telford, TF1 6TF, UK: mbillingham@doctors.org.uk

Received 28 May 2012 Revised 17 November 2012 Accepted 20 November 2012 Published Online First 22 January 2013
To cite: Billingham MJ, Billingham S-J. BMJ Supportive \& Palliative Care 2013;3:144-154.

\title{
Congruence between preferred and actual place of death according to the presence of malignant or non-malignant disease: a systematic review and meta-analysis
}

\author{
Matthew James Billingham, ${ }^{1,2}$ Sarah-Jane Billingham ${ }^{3}$
}

\section{ABSTRACT}

Objectives Achieving congruence between preferred and actual place of death is increasingly being used as a quantifiable indicator of the effectiveness of palliative care services. Current secular trends indicate a reversal of the institutionalisation of death, but this has been more pronounced in patients with malignant disease. As such, this study was conducted to evaluate whether the preservation of autonomy has been addressed equitably for cancer and non-cancer populations.

Methods A systematic review and meta-analysis was performed in line with recommendations from Preferred Reporting Items for Systematic Reviews and Meta-Analyses and the Meta-analysis Of Observational Studies in Epidemiology (MOOSE) guidelines. A comprehensive computerised search of 14 databases, supplemented by a manual review of the literature was performed for all peer-reviewed publications, from 1980 to the present, identifying rates of incongruence for cancer and/or non-cancer cohorts. Aggregation of outcomes was performed using a random effects model.

Results A total of 26 articles were identified that met the search and inclusion criteria. A non-cancer diagnosis was found to significantly increase the incidence of incongruence with a weighted risk ratio of $1.23(95 \% \mathrm{Cl} 1.01$ to $1.49, \mathrm{p}=0.04)$ and this disparity appears to have increased since 2004. A moderate degree of heterogeneity was noted $\left(I^{2}=62 \%\right)$.

Conclusions These findings pose a significant challenge, as a 'good' death has been closely matched to achieving patients' wishes, including preferred place of death. Despite increasing awareness that those dying from chronic nonmalignant diseases have extensive palliative care needs, much is still to be done to preserve their autonomy and reduce powerlessness in the face of death.

\section{INTRODUCTION}

Debates relating to what constitutes a 'good death' often place particular emphasis on being pain free, peaceful and dignified. ${ }^{1}$ Since 2004 , achievement of preferred place of death for terminally ill individuals has also increasingly been used as a quantifiable indicator of the effectiveness of palliative care services. ${ }^{2}$

To date, despite enormous efforts to resolve deficiencies in fulfilling the wishes of terminally ill individuals, suggestions of low levels of congruence, defined as the 'agreement between a patient's stated preferred place of death and actual place of death' remain. ${ }^{3}$ While population studies have indicated that the majority of people prefer to die at home, secular trends towards an institutionalised death have been reported in many countries. $^{4}$

Encouragingly, evidence of a reversal of this trend has been demonstrated in the UK, largely due to the commitment and dedication of the palliative movement. ${ }^{4}$ However, palliative care services have traditionally concentrated on the needs of a minority of people with advanced incurable disease, typically cancer patients ${ }^{5}$ and unfortunately, this reversal has been noted to be significantly more pronounced in cancer than in non-cancer deaths. ${ }^{4}$

Although home represents the preferred place of death for the majority of terminally ill individuals, ${ }^{3}$ this wish is certainly not shared by all. Inconsistent trend reversal alone, therefore, is unable to confirm whether autonomy and choice is being addressed inequitably for cancer and non-cancer groups. 
Current observations do, however, seem to imply lack of choice at end-of-life, ${ }^{3}$ especially for patients with non-malignant disease, yet evidence of an association between disease process and incidence of incongruence, has not been systematically compared across studies to date. As such, this multinational systematic review and meta-analysis was conducted to evaluate whether those who die of malignancy are being disproportionately 'singled out for deluxe dying' ${ }^{6}$ in locations of their choice.

\section{METHODS}

\section{Literature search}

A comprehensive literature review was conducted between April and October 2012. All available electronic databases including the BNI, CINAHL, Cochrane Library, EMBASE, AMED, HMIC, PsycINFO, Intute: Health \& Life Sciences, MEDLINE, PubMed, BIOSIS Previews, ISI Web of Knowledge, ISI Web of Science and IgentaConnect, were systematically searched, between 1980 to the present for all English and non-English language articles.

The following Medical Subject Heading (MeSH) terms and free text terms were used: (choice OR preference OR decision OR wish) AND (palliative OR terminal OR end-of-life OR hospice OR elderly OR aged) AND (care or death) AND (place OR location OR site). No search limits were used and further searches were performed to obtain articles from the bibliographical sections of the studies identified from the original search. The titles and/or abstracts of all identified studies were reviewed and full manuscripts obtained for those that appeared potentially relevant.

\section{Selection criteria}

Articles were included if they reported original data quantitatively comparing adult patients' preferred place of death and actual place of death. These articles were included if they assessed cancer only groups, non-cancer only groups or mixed cancer and noncancer populations where sufficient information was provided to determine congruence for both groups separately. All articles were included irrespective of whether congruence was the primary or secondary purpose of the study.

Articles that did not report either congruence, or both patients' preferred place of death and actual place of death, were excluded. Studies eliciting preferred place of care rather than place of death were also excluded, as these have been shown to be different outcomes. ${ }^{7}$ Studies were, however, included if the studies assessed preferred place of final care, as this was felt to accurately reflect preferred place of death.

Studies focusing exclusively on nursing home patients and patients receiving non-uniformly available visiting nurse station support in their homes, were excluded. Studies exclusively examining children and those studies examining association of place of death with subsequent events (including bereavement problems) rather than pre-existing preference, reviews, duplicate data, comments, case histories, qualitative studies and unpublished material were also excluded.

\section{Data extraction}

Standardised forms were used for reviewing each article and the following data was extracted and systematically entered into a matrix table: source population, study design, patient diagnosis, grade of study, congruence by location, overall congruence and incongruence rates for cancer and/or non-cancer patient groups where applicable.

Overall congruence for each study and each cancer and non-cancer cohort was determined by dividing the number of subjects with met preferences for any location of death by the number of subjects with a recorded preferred place of death while incongruence was determined by dividing the number of subjects with unmet preferences for any location of death by the number of subjects with a recorded preferred place of death. This allowed for exclusion of those patients who were unwilling or unable to express a preference for place of death, resulting in potential alteration to the reported congruence values of the included studies. MJB extracted the data from the papers and SJB assessed a 35\% random sample of papers to ensure an accurate data extraction process.

\section{Quality assessment and grading of evidence}

All studies included in the analysis were graded using quality measures defined previously. ${ }^{3}$ These criteria were as follows: grade A (strong evidence): longitudinal study with standardised systematic and predefined assessment of preference for place of terminal care or death, more than $80 \%$ response rate (if survey study), patients not limited to those within one service, (eg, one palliative care team); grade B (moderate evidence): longitudinal study that does not meet criteria for A, cross-sectional, observational, or retrospective study, more than $60 \%$ response rate (if survey study), standardised and systematic assessment of preference for place of terminal care or death; and grade C (weak evidence): response rate less than $60 \%$ or not given (if survey study), or inconsistent assessment of preferences.

\section{Analysis}

Meta-analysis was performed in line with recommendations from the Preferred Reporting Items for Systematic Reviews and Meta-Analyses and Meta-analysis Of Observational Studies in Epidemiology (MOOSE) initiative guidelines.

\section{Assessment for association}

In order to analyse rates of incongruence for the cancer and non-cancer groups, statistical analysis was carried out using weighted risk ratio estimates, comparing the 
non-cancer group with the reference (cancer) group using a random effects model. A weighted risk ratio less than 1 was set to favour the non-cancer group while a weighted relative risk greater than 1 was set to favour the cancer group. The point estimate of the weighted relative risk estimate was considered statistically significant at the $\mathrm{p}<0.05$ level if the $95 \%$ CI did not include the value 1 . All statistical analysis was conducted with Review Manager V.5.1 (Cochrane Collaboration, Oxford, UK).

\section{Heterogeneity}

A measure of the extent of variation among the effects observed in different studies was incorporated to assess the generalisability of the combined results. Statistical quantification of inconsistency was performed using Cochran's $Q$ and $I^{2}$.

\section{RESULTS}

Overall, 718 unique citations were identified and a flow chart of the inclusion and exclusion of studies is shown in figure 1. Studies presenting inappropriate subject matter $(n=596)$, reporting preferred place of care, not place of death $(n=4)$, exclusively examining nursing home $(\mathrm{n}=1)$, home with visiting nurse station support $(\mathrm{n}=1)$, paediatric populations $(\mathrm{n}=3)$ and descriptive studies of preference $(n=3)$ were excluded. Studies presenting duplicate data $(n=2)$ and articles that did not report either congruence, or patients' preferred place of death and actual place of death concurrently, for a cancer and/or a non-cancer cohort, were also excluded after close inspection of the full article $(n=82)$.

\section{Characteristics of studies}

Twenty-six articles met the inclusion criteria. ${ }^{8-33}$ Only four studies provided comparative data for a cancer and non-cancer cohort with two studies focusing on congruence specifically for separate cancer and non-cancer cohorts $^{13} \quad 33$ and the remaining studies examining preferences as a factor associated with place of death. ${ }^{8}{ }^{15}$ Seventeen studies provided non-comparative data for cancer patients only, ${ }^{9-12} 14 \quad 16 \quad 18-2023252628-32$ while five studies analysed congruence for non-cancer patients exclusively. ${ }^{172122} 2427$

A $\kappa$ statistic of 0.77 was noted between the study evaluations by $\mathrm{MJB}$ and SJB, indicating substantial interobserver agreement. Unfortunately, inadequate information was provided to determine $\kappa$ statistics for agreement between preferred and actual death in 21 studies, ${ }^{8-18} 20-273233$ precluding further use of the statistic for cross-study comparison in this review.

Of the included studies, twelve were from the

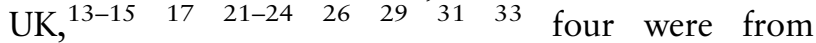
the USA $^{10}{ }^{20} \quad 27 \quad 28$ and two were from the Netherlands. ${ }^{8}{ }^{32}$ Spain, ${ }^{9}$ Italy, ${ }^{11}$ Denmark, ${ }^{12}$ Japan, ${ }^{16}$ Sweden, ${ }^{18}$ Taiwan, ${ }^{19}$ Belgium ${ }^{25}$ and Ireland ${ }^{30}$ each had one study included. Overall, a non-European nationality was associated with a statistically significant increased risk of incongruence between preferred and actual place of death. The non-weighted risk ratio of incongruence for non-European patients (39.7\%) compared with European patients (31.4\%) was 1.26 (95\% CI 1.19 to $1.34, \mathrm{p}<0.0001)$.

Table 1 summarises the study descriptions, source populations, study design, patient diagnosis, the rates of incongruence of the cancer and non-cancer cohorts and grading of each study.

Four prospective studies were graded A level. $^{28} 293133$ Eleven studies were graded B level because of retrospective design, single-service populations, or response rates less than 80\%.8 ${ }^{8} 111314171922262730$ Eleven studies were also graded $\mathrm{C}$ level because of response rates less than $60 \%$ or inconsistent assessment of preferences. $^{10} 12151618202123-2532$

The grade B and C studies were noted to produce a significantly greater mean overall congruence $(68.2 \%$ and $62.3 \%$ respectively) than the grade A studies

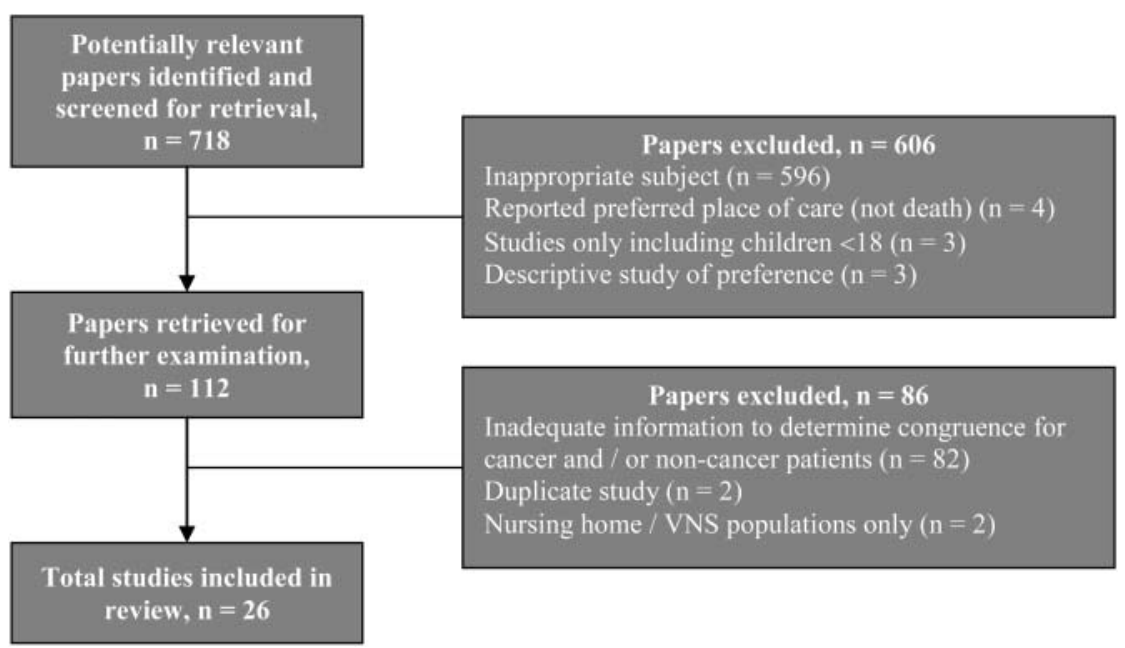

Figure 1 Study selection process. 


\begin{tabular}{|c|c|c|c|c|c|c|}
\hline Study description & Study design & $\begin{array}{l}\text { Patient } \\
\text { diagnosis }\end{array}$ & $\begin{array}{l}\text { Proportion of } \\
\text { patients } \\
\text { preferring home } \\
\text { death }(\%)\end{array}$ & $\begin{array}{l}\text { Cancer cohort congruence by } \\
\text { location (where available), } \\
\text { overall congruence* and } \\
\text { overall incongruencet }(\%)\end{array}$ & $\begin{array}{l}\text { Non-cancer cohort congruence } \\
\text { by location (where available), } \\
\text { overall congruence* and } \\
\text { overall incongruencet }(\%)\end{array}$ & Grading of study \\
\hline $\begin{array}{l}\text { Abarshi et al }(2011)^{8} 2008 \text {, the } \\
\text { Netherlands Dutch sentinel } \\
\text { network of general practitioners, } \\
n=252\end{array}$ & $\begin{array}{l}\text { 1-year follow-back study via a general } \\
\text { practitioner surveillance network in the } \\
\text { Netherlands }\end{array}$ & $38 \%$ cancer & $\mathrm{N} / \mathrm{A}$ & $\begin{array}{l}\text { Overall: } 32 / 72(44.4 \%) \ddagger \\
\text { Incongruence: } 40 / 72(55.6 \%)\end{array}$ & $\begin{array}{l}\text { Overall: 18/93 (19.4\%)‡ } \\
\text { Incongruence: } 75 / 93(80.6 \%)\end{array}$ & $\begin{array}{l}\text { B, } 65.5 \% \text { preferred place } \\
\text { of death response rate, } \\
\text { retrospective }\end{array}$ \\
\hline $\begin{array}{l}\text { Alonso-Babarro et al }(2011)^{9} \\
2004 \text { to 2006, Spain (Madrid) } \\
\text { region health areas palliative } \\
\text { home care teams, } n=380\end{array}$ & $\begin{array}{l}\text { 3-year prospective observational cohort } \\
\text { study }\end{array}$ & $100 \%$ cancer & 79.8 & $\begin{array}{l}\text { Home: } 161 / 182(88 \%) \\
\text { Other: } 42 / 46(91 \%) \\
\text { Overall: } 203 / 228(89.0 \%) \\
\text { Incongruence: } 25 / 228(11.0 \%)\end{array}$ & & $\begin{array}{l}\text { B, } 60.0 \% \text { preferred place } \\
\text { of death response rate }\end{array}$ \\
\hline $\begin{array}{l}\text { Bakitas et al }(2008)^{10} 1999 \text { to } \\
\text { 2001, USA outpatient palliative } \\
\text { care, } n=268\end{array}$ & $\begin{array}{l}\text { Family proxy respondents of deceased } \\
\text { patients with breast, gastrointestinal or } \\
\text { lung cancer who had participated in } \\
\text { Project ENABLE§ }\end{array}$ & $100 \%$ cancer & $\mathrm{N} / \mathrm{A}$ & $\begin{array}{l}\text { Overall: } 83 / 125(66.4 \%) \ddagger \\
\text { Incongruence: } 42 / 125(33.6 \%)\end{array}$ & & $\begin{array}{l}\text { C, } 46.6 \% \text { preferred place } \\
\text { of death response rate }\end{array}$ \\
\hline $\begin{array}{l}\text { Beccaro et al }(2006)^{11} 2000 \text { to } \\
2002 \text {, Italy population survey of } \\
\text { patients who died of cancer, } \\
n=1271\end{array}$ & $\begin{array}{l}\text { Retrospective national mortality } \\
\text { follow-back quantitative caregiver survey } \\
\text { (ISDOC)ף }\end{array}$ & $100 \%$ cancer & 93.5 & $\begin{array}{l}\text { Home: } 614 / 938(65 \%) \\
\text { Hospital: } 45 / 49(92 \%) \\
\text { Hospice: } 1 / 2(50 \%) \\
\text { NH: } 13 / 14(93 \%) \\
\text { Overall: } 673 / 1003(67.1 \%) \\
\text { Incongruence: } 330 / 1003(32.9 \%)\end{array}$ & & $\begin{array}{l}\text { B, } 78.9 \% \text { preferred place } \\
\text { of death response rate, } \\
\text { retrospective }\end{array}$ \\
\hline $\begin{array}{l}\text { Brogaard et al }(2012)^{12} 2008 \text { to } \\
\text { 2009, Denmark (former Aarhus } \\
\text { County), } n=96\end{array}$ & $\begin{array}{l}\text { Prospective serial interview and } \\
\text { questionnaire study from three specialist } \\
\text { palliative care teams }\end{array}$ & $100 \%$ cancer & 68.8 & $\begin{array}{l}\text { Home: } 16 / 33(48 \%) \\
\text { Hospital: } 0 / 2(0 \%) \\
\text { Hospice: } 5 / 12(42 \%) \\
\text { NH: } 0 / 1(0 \%) \\
\text { Overall: } 21 / 48(43.8 \%) \\
\text { Incongruence: } 27 / 48(56.2 \%)\end{array}$ & & $\begin{array}{l}\text { C, } 50 \% \text { preferred place } \\
\text { of death response rate }\end{array}$ \\
\hline $\begin{array}{l}\text { Capel et al }(2012)^{13} 2009 \text { to } \\
2010 \text {, Wales regional (Cardiff) } \\
\text { specialist community palliative } \\
\text { care service, } n=788\end{array}$ & $\begin{array}{l}\text { Prospective observation with patient } \\
\text { interview to determine preferences }\end{array}$ & $93 \%$ cancer & 67.3 & $\begin{array}{l}\text { Home: } 236 / 348(68 \%) \\
\text { Hospital: } 10 / 12(83 \%) \\
\text { Hospice: } 90 / 108(83 \%) \\
\text { NH: } 27 / 29(93 \%) \\
\text { Other: } 12 / 20(60 \%) \\
\text { Overall: } 375 / 517(72.5 \%) \\
\text { Incongruence: } 142 / 517(27.5 \%)\end{array}$ & $\begin{array}{l}\text { Home: } 27 / 32(84 \%) \\
\text { Hospital: } 2 / 2(100 \%) \\
\text { Hospice: } 3 / 6(50 \%) \\
\text { NH: } 6 / 7(86 \%) \\
\text { Other: } 1 / 1(100 \%) \\
\text { Overall: } 39 / 48(81.2 \%) \\
\text { Incongruence: } 9 / 48(18.8 \%)\end{array}$ & $\begin{array}{l}\text { B, } 71.7 \% \text { preferred place } \\
\text { of death response rate }\end{array}$ \\
\hline $\begin{array}{l}\text { Carroll }(1998)^{14} 1995 \text { to } 1996, \\
\text { Scotland Aberdeen, } n=34\end{array}$ & $\begin{array}{l}\text { 1-year prospective audit with general } \\
\text { practitioner, community nurse, relatives } \\
\text { and/or carer interview }\end{array}$ & $100 \%$ cancer & 76.7 & $\begin{array}{l}\text { Home: } 16 / 23(70 \%) \\
\text { Hospital: } 0 / 0(0 \%) \\
\text { Hospice: } 1 / 1(100 \%) \\
\text { NH: } 6 / 6(100 \%) \\
\text { Overall: } 23 / 30(76.7 \%) \\
\text { Incongruence: } 7 / 30(23.3 \%)\end{array}$ & & $\begin{array}{l}\text { B, } 88.2 \% \text { preferred place } \\
\text { of death response rate, } \\
\text { single service }\end{array}$ \\
\hline
\end{tabular}


Fukui et al (2003) ${ }^{16}$ 2001, Japan Nationwide study, questionnaire survey 259 home care agencies, $n=528$ response from home care agencies selected from a list of the National Association for Home-visit Nursing Care (NAHNC)

Guthrie et al (1996) $)^{17} 1994$ England St Stephen's clinic London, $n=124$

Gyllenhammar et al (2003) 1999, Sweden palliative home care teams, $n=221$

Hsieh et al (2007) $)^{19} 2007$, Taiwan palliative care hospital unit, $\mathrm{n}=46$

Jeurkar et al (2012) 2008 to 2011, USA (three hospice programs in Florida,

Pennsylvania, and Wisconsin),

$n=7391$

Johnson et al (2009) 212006 to 2007, England (Bradford), $n=86$

Johnson et al $(2012)^{22} 2009$ to 2010, England (Scarborough and Bradford/Airedale), $n=126$

fostionnaire for caregivers in five palliative home care teams

Prospective cohort survey of patient and family, follow-up bereavement interview

3-year electronic health record-based retrospective cohort study cardiology palliative care teams, patient cardiology palliative care teams, patient interview y design

Prospective observation with patient interview to determine preferences

6-month retrospective key worker interview

1-year prospective study by two integrated interview (one care team data excluded) t† 1 -year prospective study by two integrated

$100 \%$ cancer $\quad 88.5$
$0 \%$ cancer

$100 \%$ HIV

Proportion of

patients

diagnosis death $(\%)$

97\% cancer

53.3

$00 \%$ cancer

79.4

Cancer cohort congruence by ocation (where available)

overall congruence* and

overall incongruencet $(\%)$

Home: 38/48 (79\%)

Hospital: $11 / 11(100 \%)$

Hospice: $13 / 26(50 \%)$

$\mathrm{NH}: 0 / 0(0 \%)$

Overall: $62 / 85(72.9 \%)$

Incongruence: 23/85 (27.1\%)

For spontaneously expressed

home preference only: Overall:

$288 / 419(68.7 \%)^{* *}$

Incongruence: 131/419 (31.3\%)

$100 \%$ cancer $\quad 37.1$

$100 \%$ cancer $\quad 73.9$

$0 \%$ cancer

$100 \%$ heart

failure

$0 \%$ cancer

$100 \%$ heart

failure

For spontaneously expressed

home preference only: Overall: 63 /

$82(76.8 \%)^{* *}$

Incongruence: 19/82 (23.2\%)

Overall: 20/46 (43.5\%)‡

ncongruence: 26/46 (56.5\%)

Home: $1877 / 3153(60 \%)$

Other: $266 / 408(65 \%)$

Overall: 2143/3561 (60.2\%)

Incongruence: 1418/3561

$(39.8 \%)$

N/A
Non-cancer cohort congruence

by location (where available).

overall congruence* and

overall incongruencet $(\%) \quad$ Grading of study

Home: 0/0 (0\%)

Hospital: $2 / 2(100 \%)$

Hospice: 0/0 (0\%)

$\mathrm{NH}: 0 / 3(0 \%)$

Overall: $2 / 5(40.0 \%)$

Incongruence: $3 / 5(60.0 \%)$

C, $56.3 \%$ preferred place
of death response rate,

single service

preference not

assessed consistently,

congruence for all

locations of death not

assessed

Home: 35/38 (92\%)

Hospital: 15/58 (26\%)

Hospice: $22 / 25(88 \%)$

Other: $1 / 3(33 \%)$

B, 100\% preferred place of death response rate

retrospective, single

service

verall: 73/124 (58.9\%)

Overall: 22/34 (64.7\%) ‡

Incongruence: 12/34 (35.3\%)

C, preference not assessed consistently, congruence for all

locations of death not assessed

B, $100 \%$ preferred place of death response rate, single service

C, $42.7 \%$ preferred place of death response rate,

retrospective

C, 39.5\% preferred place of death response rate

Home: 35/69 (51\%)

Hospital: 4/4 (100\%)

Hospice: 12/12 (100\%)

Overall: 51/85 (60.0\%)

Incongruence: $34 / 85$ (40.0\%)
B, $67.4 \%$ preferred place of death response rate 


\begin{tabular}{|c|c|c|c|c|c|c|}
\hline Study description & Study design & $\begin{array}{l}\text { Patient } \\
\text { diagnosis }\end{array}$ & $\begin{array}{l}\text { Proportion of } \\
\text { patients } \\
\text { preferring home } \\
\text { death }(\%)\end{array}$ & $\begin{array}{l}\text { Cancer cohort congruence by } \\
\text { location (where available), } \\
\text { overall congruence* and } \\
\text { overall incongruencet }(\%)\end{array}$ & $\begin{array}{l}\text { Non-cancer cohort congruence } \\
\text { by location (where available), } \\
\text { overall congruence* and } \\
\text { overall incongruencet }(\%)\end{array}$ & Grading of study \\
\hline $\begin{array}{l}\text { Karlsen et al }(1998)^{23} 1995 \text { to } \\
\text { 1996, England cancer death } \\
\text { certificates, } n=229\end{array}$ & Random sample of cancer deaths & $100 \%$ cancer & 73.6 & $\begin{array}{l}\text { Home: } 37 / 64(58 \%) \\
\text { Other: } 18 / 23(78 \%) \\
\text { Overall: } 55 / 87(63.2 \%) \\
\text { Incongruence: } 32 / 87(36.8 \%)\end{array}$ & & $\begin{array}{l}\text { C, 38.0\% preferred place } \\
\text { of death response rate }\end{array}$ \\
\hline $\begin{array}{l}\text { McKeown et al }(2008)^{24} 2004 \\
\text { to 2006, England (Liverpool), } \\
n=29\end{array}$ & $\begin{array}{l}\text { 2-year retrospective audit assessing current } \\
\text { referral practice for patients with } \\
\text { end-stage renal failure }\end{array}$ & $\begin{array}{l}0 \% \text { cancer } \\
100 \% \text { renal } \\
\text { failure }\end{array}$ & 50.0 & & $\begin{array}{l}\text { Home: } 3 / 7(43 \%) \\
\text { Hospital: } 2 / 2(100 \%) \\
\text { Hospice: } 0 / 4(0 \%) \\
\text { NH: 0/1 }(0 \%) \\
\text { Overall: } 5 / 14(35.7 \%) \\
\text { Incongruence: } 9 / 14(64.3 \%)\end{array}$ & $\begin{array}{l}\text { C, } 48.3 \% \text { preferred place } \\
\text { of death response rate }\end{array}$ \\
\hline $\begin{array}{l}\text { Meeussen et al }(2011)^{25} 2008 \text {, } \\
\text { Belgium and the Netherlands } \\
\text { nationwide sentinel networks of } \\
\text { general practitioners, } n=422\end{array}$ & $\begin{array}{l}\text { 1-year nationally representative } \\
\text { retrospective follow-back study }\end{array}$ & $100 \%$ cancer & $\mathrm{N} / \mathrm{A}$ & $\begin{array}{l}\text { Overall: } 167 / 219(76.3 \%) \ddagger \\
\text { Incongruence: } 52 / 219(23.7 \%)\end{array}$ & & $\begin{array}{l}\text { C, } 51.9 \% \text { preferred place } \\
\text { of death response rate, } \\
\text { retrospective }\end{array}$ \\
\hline $\begin{array}{l}\text { Scholes and Allan (2012) } \\
\text { 2012, England (Mount Vernon } \\
\text { Cancer Network), } n=708\end{array}$ & $\begin{array}{l}\text { 3-month network-wide audit of } \\
\text { consecutive patients known to specialist } \\
\text { palliative care teams }\end{array}$ & $100 \%$ cancer & 43.2 & $\begin{array}{l}\text { Overall: } 349 / 426(81.9 \%) \ddagger \\
\text { Incongruence: } 77 / 426(18.1 \%)\end{array}$ & & $\begin{array}{l}\text { B, } 60.2 \% \text { preferred place } \\
\text { of death response rate, } \\
\text { retrospective, single } \\
\text { service }\end{array}$ \\
\hline $\begin{array}{l}\text { Shega et al }(2008)^{27} 2000 \text { to } \\
2001 \text {, USA Geriatric clinic, } \\
n=135\end{array}$ & $\begin{array}{l}\text { 2-year prospective study with } \\
\text { community-dwelling dementia patient } \\
\text { caregiver interviews, University of Chicago } \\
\text { geriatrics clinics }\end{array}$ & $\begin{array}{l}0 \% \text { cancer } \\
100 \% \\
\text { dementia }\end{array}$ & $\mathrm{N} / \mathrm{A}$ & & $\begin{array}{l}\text { Overall: 69/104 (66.3\%)‡ } \\
\text { Incongruence: } 35 / 104(33.7 \%)\end{array}$ & $\begin{array}{l}\text { B, } 77.0 \% \text { preferred place } \\
\text { of death response rate, } \\
\text { single service }\end{array}$ \\
\hline $\begin{array}{l}\text { Tang and McCorkle }(2003)^{28} \\
2001 \text { to } 2002 \text {, USA Connecticut, } \\
\text { six study sites, terminal cancer } \\
\text { patients, } n=127\end{array}$ & $\begin{array}{l}\text { 1-year prospective observation with patient } \\
\text { interview }\end{array}$ & $100 \%$ cancer & 87.4 & $\begin{array}{l}\text { Home: } 25 / 111(23 \%) \\
\text { Hospital: } 3 / 3(100 \%) \\
\text { Hospice: } 9 / 10(90 \%) \\
\text { NH: } 1 / 3(33 \%) \\
\text { Overall: } 38 / 127(29.9 \%) \\
\text { Incongruence: } 89 / 127(70.1 \%)\end{array}$ & & $\begin{array}{l}\text { A, } 100 \% \text { preferred place } \\
\text { of death response rate }\end{array}$ \\
\hline $\begin{array}{l}\text { Thomas et al }(2004)^{29} 2000 \text { to } \\
2002, \text { England terminal cancer } \\
\text { patients, } n=41\end{array}$ & $\begin{array}{l}\text { 2-year prospective longitudinal observation } \\
\text { with serial cancer patient interviews }\end{array}$ & $100 \%$ cancer & 29.4 & $\begin{array}{l}\text { Home: } 6 / 10(60 \%) \\
\text { Hospice: } 4 / 4(100 \%) \\
\text { Overall: } 10 / 14(71.4 \%) \\
\text { Incongruence: } 4 / 14(28.6 \%)\end{array}$ & & $\begin{array}{l}\text { A, } 82.9 \% \text { preferred place } \\
\text { of death response rate }\end{array}$ \\
\hline $\begin{array}{l}\text { Tiernan et al }(2002)^{30} 2002 \text {, } \\
\text { Ireland home care team, } n=191\end{array}$ & Prospective observational cohort study & $100 \%$ cancer & 81.8 & $\begin{array}{l}\text { Home: } 57 / 121(47 \%) \\
\text { Hospital: } 2 / 5(40 \%) \\
\text { Hospice: } 10 / 17(59 \%) \\
\mathrm{NH}: 4 / 5(80 \%) \\
\text { Overall: } 73 / 148(49.3 \%) \\
\text { Incongruence: } 75 / 148(50.7 \%)\end{array}$ & & $\begin{array}{l}\text { B, } 77.5 \% \text { preferred place } \\
\text { of death response rate, } \\
\text { single service }\end{array}$ \\
\hline
\end{tabular}




\begin{tabular}{|c|c|c|c|c|c|c|}
\hline Study description & Study design & $\begin{array}{l}\text { Patient } \\
\text { diagnosis }\end{array}$ & $\begin{array}{l}\text { Proportion of } \\
\text { patients } \\
\text { preferring home } \\
\text { death }(\%)\end{array}$ & $\begin{array}{l}\text { Cancer cohort congruence by } \\
\text { location (where available), } \\
\text { overall congruence* and } \\
\text { overall incongruencet }(\%)\end{array}$ & $\begin{array}{l}\text { Non-cancer cohort congruence } \\
\text { by location (where available), } \\
\text { overall congruence* and } \\
\text { overall incongruencet }(\%)\end{array}$ & Grading of study \\
\hline $\begin{array}{l}\text { Townsend et al }(1990)^{31} 1986 \\
\text { to 1987, England hospital/ } \\
\text { community terminal cancer } \\
\text { patients, } n=70\end{array}$ & $\begin{array}{l}\text { 1-year prospective random-sample } \\
\text { longitudinal observation, serial patient } \\
\text { interviews }\end{array}$ & $100 \%$ cancer & 30.5 & $\begin{array}{l}\text { Home: } 17 / 18(94 \%) \\
\text { Hospital: } 12 / 32(38 \%) \\
\text { Hospice: } 7 / 9(78 \%) \\
\text { Overall: } 36 / 59(61.0 \%) \\
\text { Incongruence: } 23 / 59(39.0 \%)\end{array}$ & & $\begin{array}{l}\text { A, } 84.3 \% \text { preferred place } \\
\text { of death response rate }\end{array}$ \\
\hline $\begin{array}{l}\text { Van der Heide et al }(2007)^{32} \\
\text { 2006, the Netherlands, } n=63\end{array}$ & $\begin{array}{l}\text { Retrospective sub-sample of a cohort of } \\
\text { advanced cancer patients, interview with } \\
\text { bereaved relatives }\end{array}$ & $100 \%$ cancer & 78.1 & $\begin{array}{l}\text { Home: } 20 / 25(80 \%) \\
\text { Hospital: } 2 / 2(100 \%) \\
\text { Hospice: } 3 / 3(100 \%) \\
\text { Other: } 0 / 2(0 \%) \\
\text { Overall: } 25 / 32(78.1 \%) \\
\text { Incongruence: } 7 / 32(21.9 \%)\end{array}$ & & $\begin{array}{l}\text { C, } 50.8 \% \text { preferred place } \\
\text { of death response rate }\end{array}$ \\
\hline $\begin{array}{l}\text { Walker et al }(2010)^{33} 2007 \text { to } \\
\text { 2010, England regional (South } \\
\text { Devon) specialist community }\end{array}$ & $\begin{array}{l}\text { Prospective, longitudinal observation with } \\
\text { serial patient/carer interviews }\end{array}$ & $88 \%$ cancer & 53.7 & $\begin{array}{l}\text { Overall: } 17 / 36(47.2 \%) \ddagger \\
\text { Incongruence: } 19 / 36(52.8 \%)\end{array}$ & $\begin{array}{l}\text { Overall: } 1 / 5(20.0 \%) \ddagger \\
\text { Incongruence: } 4 / 5(80.0 \%)\end{array}$ & $\begin{array}{l}\text { A, } 100 \% \text { preferred place } \\
\text { of death response rate }\end{array}$ \\
\hline
\end{tabular}

palliative care service, $n=41$

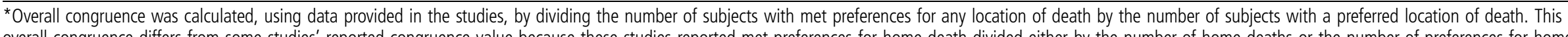

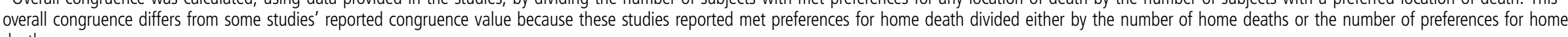
death.

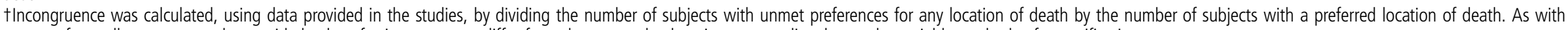
reports of overall congruence, the provided values for incongruence differ from the reported values in some studies due to the variable methods of quantification.

‡Data not provided to calculate congruence individually for all locations of death: only overall congruence information available.

§Project ENABLE (Educate, Nurture, Advise, Before Life Ends): an outpatient palliative care early intervention demonstration quality improvement project implemented at two oncology care settings.

IISDOC study (Italian Survey of the Dying of Cancer): a national mortality follow-back survey of 2000 cancer deaths.

**Percentage of patients who spontaneously expressed a wish to die at home. No other elicitation of preference was reported. Data not provided to calculate overall congruence for all locations of death.

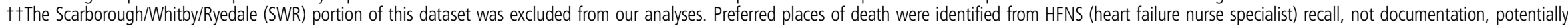
introducing recall bias. 
(42.3\%) with a wide range of overall congruence noted for each grade. Two grade $\mathrm{C}$ studies reported only home congruence. ${ }^{16}{ }^{18}$ Seven studies-two grade $\mathrm{A},{ }^{28}{ }^{33}$ three grade $\mathrm{B}^{8}{ }^{19}{ }^{30}$ and two grade $\mathrm{C},{ }^{12} 24$ reported less than $50 \%$ overall congruence.

\section{Illness factors and congruence}

Seventeen studies included cancer-only patients, ${ }^{9-12} \quad 14 \quad 16 \quad 18-20 \quad 23 \quad 25 \quad 26 \quad 28-32$ while three mixed population studies included more than $85 \%$ of patients with cancer. ${ }^{13} 1533$ These 20 studies noted a wide range of overall congruence of 30-89\%. Five studies exclusively examined non-cancer patients, ${ }^{17} \quad 21 \quad 22 \quad 24 \quad 27$ with one mixed-population study reporting on less than $40 \%$ of patients with cancer. ${ }^{8}$ These six studies also noted a wide range of overall congruence of $30-66 \%$. There was no clear correlation between levels of overall congruence and the percentage of patients with cancer diagnoses $(\mathrm{p}=0.18)$.

Analysis of incongruence according to diagnosis group (cancer or non-cancer) was based on 5036 (64.0\%) congruent and 2840 (36.0\%) incongruent deaths in 7876 participants. Overall, 232 (45.3\%) incongruent deaths occurred in 512 participants without cancer and 2608 (35.4\%) incongruent deaths occurred in 7364 participants with a cancer diagnosis. Overall, a non-cancer diagnosis was associated with a greater risk of incongruence between preferred and actual place of death (figure 2). The weighted risk ratio for incongruence for non-cancer patients compared with cancer patients was 1.23 (95\% CI 1.01 to $1.49, \mathrm{p}=0.04)$. A moderate degree of heterogeneity was observed $\left(\mathrm{I}^{2}=62 \%\right)$.

\section{Secular trends and reversal of institutionalisation of death}

Nine studies were published prior to 2004, including seven cancer-only, ${ }^{14} 161823283031$ one non-cancer only ${ }^{17}$ and one mixed cancer and non-cancer populations, ${ }^{15}$ while 17 studies were released after the achievement of preferred place of death was brought to the forefront of the medical community. ${ }^{34} 35$ Ten of these studies included only cancer partici-

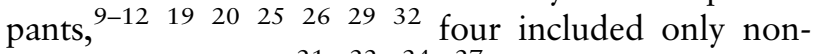
cancer participants ${ }^{21} 22 \quad 24 \quad 27$ and three included cancer and non-cancer cohorts. ${ }^{8} 1333$
In line with the aforementioned reversal of secular trends, indicating greater emphasis on meeting terminally ill patient's wishes, there was a significant overall reduction of incongruence since 2004. This improvement was significantly more pronounced for cancer patients than non-cancer patients $(\mathrm{p}=0.0095)($ table 2$)$.

\section{Preferred location of death}

Only four studies reported preferences for every patient in the sample. ${ }^{17} 192833$ In the remaining studies, 12$62 \%$ of patients did not express a preference for place of death. ${ }^{8-16} 18$ 20-27 29-32 Twenty-one studies specifically identified rates of preference for home death. In sixteen of these studies more than $50 \%$ of participants preferred home deaths, after disregarding patients without an expressed preference, ${ }^{9}$ 11-16 $19 \quad 20 \quad 22-$ 2428303233 while five studies reported on less than $45 \%$ of patients expressing a wish to die at home. ${ }^{17} 18 \quad 252931$ Overall, $79.5 \%$ of participants reporting a preference expressed a desire to die at home. Two studies reported only on $79 \%{ }^{16}$ and $37 \%{ }^{18}$ of their populations, who spontaneously expressed a preference for home death and did not elicit or report any other preference.

Analysis of incongruence according to preferred place of death (home or non-home) was based on 308 (31.0\%) incongruent deaths in 995 participants preferring a non-home death and 2150 (37.6\%) incongruent deaths, which occurred in 5721 participants preferring a home death.

Overall, a home preference was associated with a non-significantly increased risk of incongruence between preferred and actual place of death (figure 3). The weighted risk ratio for incongruence for individuals preferring a home death compared with individuals preferring a non-home death was 1.17 (95\% CI 0.84 to $1.64, \mathrm{p}=0.36$ ). A substantial degree of heterogeneity was noted $\left(\mathrm{I}^{2}=73 \%\right)$.

Overall, there was no significant difference between cancer $(62.3 \%)$ and non-cancer patients $(68.4 \%)$ in the rate of achievement of preference for those preferring a home death $(\mathrm{p}=0.095)$.

\section{DISCUSSION}

Enabling a 'good' death is closely matched to meeting patients' wishes. Although congruence has significantly improved since 2004, admissions relating to

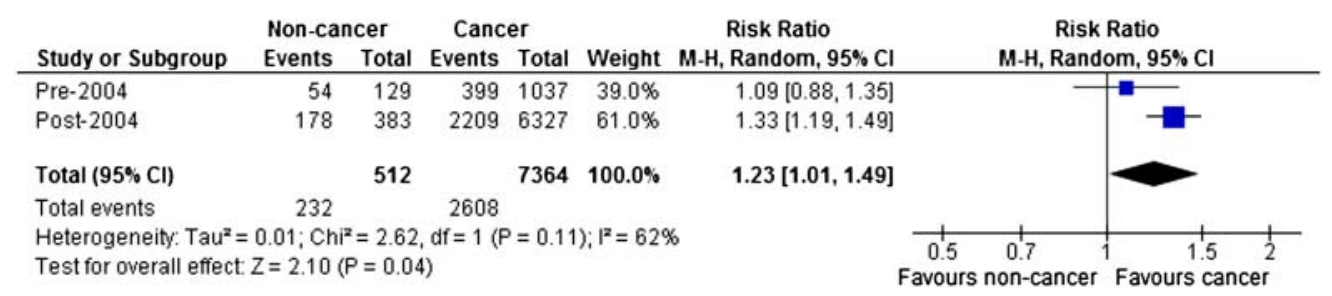

Figure 2 Incongruence risk ratio forest plot according to diagnosis. 
Table 2 Temporal trends of overall incongruence and relative risk of incongruence

\begin{tabular}{llll}
\hline Measure & Pre-2004 & Post-2004 & $\begin{array}{l}\text { Statistical } \\
\text { significance of } \\
\text { difference }\end{array}$ \\
\hline $\begin{array}{l}\text { Mean overall } \\
\text { incongruence }\end{array}$ & $38.9 \%$ & $35.6 \%$ & $\chi^{2}=30.3 p<0.0001$ \\
$\begin{array}{l}\text { Relative risk of } \\
\text { incongruence } \\
\text { (non-cancer vs cancer } \\
\text { cohort) }\end{array}$ & 1.09 & 1.33 & $\chi^{2}=6.73 p=0.0095$ \\
\hline
\end{tabular}

deficiencies in provision of choice at end-of-life have, sadly, become commonplace, especially in patients with non-malignant disease. In line with these admissions, this pooled analysis of 7876 participants from 26 observational studies has demonstrated that a noncancer diagnosis is associated with a statistically significant $23 \%$ increase in the incidence of incongruence $(p=0.04)$ and disconcertingly for non-cancer patients, this trend has become more pronounced since 2004.

Recent debate on the provision of palliative care as an international human right has included the assertion that there should be equity of access to services for all, without discrimination. ${ }^{36}$ Few can argue with the merit of this objective, yet as evident from the findings of this study few can deny the presence of serious inequalities according to patient diagnosis.

The first major challenge is to enhance recognition of terminal illness. The diagnosis of dying is often made too late, however, approximately two-thirds of all deaths are non-sudden and protracted and can be anticipated. ${ }^{37}$ Early recognition of impending death allows for better management of dying patients, although it is widely appreciated that this is more challenging for noncancer patients, whose illness trajectories are relatively more complicated and less predictable. ${ }^{8}$ Development of simple, well-validated prognostic models with good calibration and reliability may aid this process.

The second major challenge is to enhance investigation of preference. The $56.5 \%$ completion rate of preference from the 26 studies is disconcerting. In part this may be due to patient unwillingness and inability to participate in discussion but may also indicate a lack of ascertainment of preference for a considerable willing and able proportion. Having cancer, as opposed to other diagnoses, has been positively associated with increased discussion of end-of-life issues ${ }^{38}$ and awareness of preferred place of death ${ }^{8}$ and it will come as no surprise that patients who receive more healthcare contact and have their preferences assessed tended to die more often in a preferred place. ${ }^{39}$ In non-malignant disease the lack of defined trajectories may leave those who believed they had more time to consider their options unable to express a preference, potentially escalated by healthcare staff insecurities in discussion and management of non-malignant disease. ${ }^{40}$

The third major challenge is to enhance provision of care. In recent general population studies of preference, $50-90 \%$ of respondents have expressed a preference to die at home. ${ }^{3}$ However, without assistance, the end-of-life stage is a crucial testing time, especially when the decedent is at home. ${ }^{41}$ If strong support is not readily available some caregivers will be unable to bear the strain and unplanned admission may be necessary. These crises can be successfully avoided if families are well prepared and support is readily available. ${ }^{41}$

Although the goal of reducing inequity and providing care for patients on the basis of need rather than diagnosis is widely acknowledged ${ }^{36}$ given the low proportion of non-cancer patients managed by palliative care teams in the UK, sufficient clinical exposure to bridge these deficiencies is unlikely to be rapidly forthcoming.

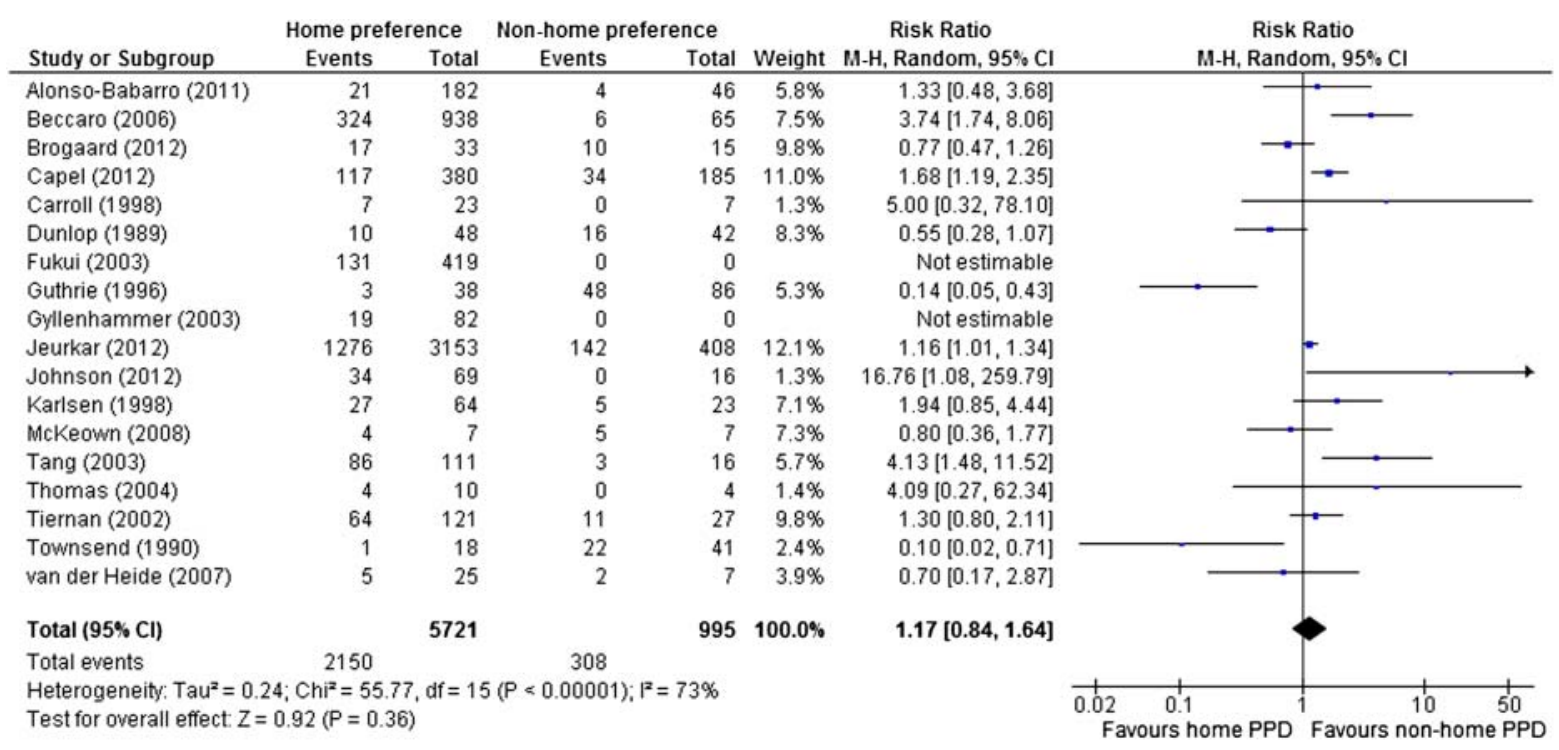

Figure 3 Incongruence risk ratio forest plot by preferred place of death. 
Instead, measures to improve recognition of the terminal phase, investigation of individual preferences and the provision of services should be opened up to non-palliative care specialist clinicians, groups currently caring for non-cancer patients and health service commissioners. These potential 'agencies for change' may possess important insights into patient needs, input into the management of experiences and deficiencies, and perhaps assist in the development of innovative services to preserve autonomy.

\section{Limitations}

Although the Cochran $\mathrm{Q}$ test does not demonstrate significant heterogeneity $(\mathrm{p}=0.11)$, the $\mathrm{I}^{2}$ value $(62 \%)$ does suggest a moderate degree of inconsistency of individual study findings in relation to the primary outcome measure. A substantial degree of heterogeneity $\left(\mathrm{I}^{2}=73 \%, \mathrm{p}<0.00001\right)$ was also observed in relation to the differing incongruence rates according to preferred place of death.

Some of this inconsistency may be explained by the constitution of the cancer and non-cancer cohorts. Patients with haematological malignancy, for example, have been shown to be more likely to die in hospital than other cancer diagnoses, ${ }^{42}$ and patients with colorectal carcinoma have been shown to be more likely to die at home. ${ }^{43}$ Therefore, it is plausible that patients with certain types of cancer may be more or less likely to have their end-of-life preferences achieved and that if there are inter-study systematic differences of cancer type, this may be responsible for some of the heterogeneity observed.

No randomised controlled trials were available for inclusion and most studies included were small and observational, with incomplete assessment of preference within a majority of studies. Smaller studies are often conducted and analysed with less methodological rigour than larger studies and in observational studies confounding variables may negate the findings. This confounding effect is compounded by the more comprehensive approach used, which allowed for inclusion of non-comparative studies exclusively studying cancer or non-cancer participants. In addition, the range of nationality of the included studies, where differing end-of-life care provisions and policies exist, heightens this confounding effect.

Selection bias is also a significant drawback of the included studies and therefore this meta-analysis. Population studies have suggested that only a minority of all dying patients receive specialist palliative care management. ${ }^{44}$ Since involvement of these teams has been associated with increased congruence across many studies, ${ }^{32845}$ given that most of the participants of the included studies were under the care of such specialists, this limits the generalisability. In addition, surveys have indicated that well over $95 \%$ of all patients seen within specialist palliative care services have cancer. ${ }^{5}$ In comparison, given that approximately $7 \%$ of all participants in the included studies had a non-cancer diagnosis, there is a substantial over-representation of these patients and it is plausible, therefore, that these findings are only a conservative estimate of the true effect.

\section{CONCLUSION}

In summary, this meta-analysis has demonstrated a significant association between a non-cancer diagnosis and increased incidence of incongruence between preferred and actual place of death and that this relationship is becoming more profound. Over the next decade this shift may become increasingly important, given that in Britain the overall numbers of deaths are expected to rise substantially in the near future ${ }^{4}$ and that the majority of these deaths are predicted to be attributable to a non-cancer diagnosis. ${ }^{46}$ With the resultant increase in end-of-life needs that is predicted, it is vital to develop investment plans and services that meet the requirements of ageing populations and ensure the best possible outcomes for terminally ill patients and their families wherever they wish to be and whatever their diagnosis.

Acknowledgements The authors would like to thank the helpful and friendly staff of the Douglas Macmillan Hospice and the Health Library for North Staffordshire for their inspiration and assistance. We are also grateful to Dr MS Billingham and Mrs MR Billingham for their insightful review and guidance in the preparation of the final manuscript.

Contributors $\mathrm{MJB}$ and SJB were responsible for overall conceptualisation of the paper, contributed to the design and construction of the review, performed the literature review, analysed the extracted papers with particular reference to data and statistical analysis and produced draft versions and the final version of the document. Both authors have also reviewed the content of the final document and have approved this final manuscript.

Funding None.

Competing interests None.

Provenance and peer review Not commissioned; externally peer reviewed.

\section{REFERENCES}

1 Vig EK, Pearlman RA. Good and bad dying from the perspective of terminally ill men. Arch Intern Med 2004;164:977-81.

2 Department of Health: End of Life Care Strategy. Promoting high quality care for all adults at the end of life. London, England: Department of Health, 2008.

3 Bell CL, Somogyi-Zalud E, Masaki KH. Factors associated with congruence between preferred and actual place of death. J Pain Symptom Manage 2010;39:591-604.

4 Gomes B, Calanzani N, Higginson IJ. Reversal of the British trends in place of death: time series analysis 2004-2010. Palliat Med 2012;26:102-7. 
5 Murtagh FE, Preston M, Higginson I. Patterns of dying: palliative care for no-malignant disease. Clin Med 2004;4:39-44.

6 Douglas C. For all the saints. Br Med J 1992;304:579.

7 Agar M, Currow DC, Shelby-James TM, et al. Preference for place of care and place of death in palliative care: are these different questions? Palliat Med 2008;22:787-95.

8 Abarshi EA, Echteld MA, van den Block L, et al. Recognising patients who will die in the near future: a nationwide study via the Dutch sentinel network of GPs. Br J Gen Pract 2011;61:371-8.

9 Alonso-Babarro A, Bruera E, Varela-Cerdeira M, et al. Can this patient be discharged home? Factors associated with at-home death among patients with cancer. J Clin Oncol 2011;29:1159-67.

10 Bakitas M, Ahles TA, Skalla K, et al. Proxy perspectives regarding end-of-life care for persons with cancer. Cancer 2008;112:1854-61.

11 Beccaro M, Costantini M, Giorgi Rossi P, et al. Actual and preferred place of death of cancer patients. Results from the Italian survey of the dying of cancer (ISDOC). J Epidemiol Community Health 2006;60:412-16.

12 Brogaard T, Neergaard MA, Sokolowski I, et al. Congruence between preferred and actual place of death among Danish cancer patients. Palliat Med 2012. [Epub ahead of print]

13 Capel M, Gazi T, Vout L, et al. Where do patients known to a community palliative care service die? BMJ Support Palliat Care 2012;2:43-7.

14 Carroll DS. An audit of place of death of cancer patients in a semi-rural Scottish practice. Palliat Med 1998;12:51-3.

15 Dunlop R, Davies R, Hockley J. Preferred versus actual place of death: a hospital palliative care support team experience. Palliat Med 1989;3:197-201.

16 Fukui S, Kawagoe H, Masako S, et al. Determinants of the place of death among terminally ill cancer patients under home hospice care in Japan. Palliat Med 2003;17:445-53.

17 Guthrie B, Nelson M, Gazzard B. Are people with HIV in London able to die where they plan? AIDS Care 1996;8:709-13.

18 Gyllenhammar E, Thoren-Todoulos E, Strang P, et al. Predictive factors for home deaths among cancer patients in Swedish palliative home care. Support Care Cancer 2003;11:560-7.

19 Hsieh MC, Huang MC, Lai YL, et al. Grief reactions in family caregivers of advanced cancer patients in Taiwan: relationship to place of death. Cancer Nurs 2007;30:278-84.

20 Jeurkar N, Farrington S, Craig TR, et al. Which hospice patients with cancer are able to die in the setting of their choice? Results of a retrospective cohort study. J Clin Oncol 2012;30:2783-7.

21 Johnson MJ, Parsons S, Raw J, et al. Achieving preferred place of death-is it possible for patients with chronic heart failure? Br J Cardiol 2009;16:194-6.

22 Johnson M, Nunn A, Hawkes T, et al. Planning for end-of-life care in heart failure: experience of two integrated cardiology-palliative care teams. Br J Cardiol 2012;19:71-5.

23 Karlsen S, Addington-Hall J. How do cancer patients who die at home differ from those who die elsewhere? Palliat Med 1998;12:279-86.

24 McKeown A, Agar R, Gambles M, et al. Renal failure and specialist palliative care: an assessment of current referral practice. Int J Palliat Nurs 2008;14:454-8.

25 Meeussen K, van den Block L, Echteld MA, et al. End of life care and circumstances of death in patients dying as a result of cancer in Belgium and the Netherlands: a retrospective comparative study. J Clin Oncol 2011;29:4327-34.
26 Scholes C, Allan R. Do patients referred to specialist palliative care (SPC) die where they prefer? A network-wide audit. BMJ Support Palliat Care 2012;2:A79.

27 Shega JW, Hougham GW, Stocking CB, et al. Patients dying with dementia: experience at the end of life and impact of hospice care. J Pain Symptom Manage 2008;35:499-507.

28 Tang ST, McCorkle R. Determinants of congruence between the preferred and actual place of death for terminally ill cancer patients. J Palliat Care 2003;19:230-7.

29 Thomas C, Morris SM, Clark D. Place of death: preferences among cancer patients and their carers. Soc Sci Med 2004;58:2431-44.

30 Tiernan E, O'Connor M, O'Siorain L, et al. A prospective study of preferred versus actual place of death among patients referred to a palliative care home-care service. Ir Med J 2002;95:232-5.

31 Townsend J, Frank AO, Fermont D, et al. Terminal cancer care and patients' preference for place of death: a prospective study. Br Med J 1990;301:415-17.

32 Van der Heide A, de Vogel-Voogt E, Visser AP, et al. Dying at home or in an institution: perspectives of Dutch physicians and bereaved relatives. Support Care Cancer 2007;15:1413-21.

33 Walker G, Anning P, Barton A. Preferred Place of Death: Factors influencing actual versus preferred place of death and the decision making process. 2010. http://www. dimblebycancercare.org/research/completed-research-projects (accessed 3 Apr 2012).

34 Davies E, Higginson J, eds. Palliative Care - The Solid Facts. Copenhagen: World Health Organisation Regional Office for Europe, 2004.

35 House of Commons Health Committee. Palliative care: Fourth Report of Session 2003-2004. Volume 1: Report, together with formal minutes. London: The Stationery Office Limited, 2004.

36 Burt J. Back to basics: researching equity in palliative care. Palliat Med 2012;26:5-6.

37 Gibbins J, McCoubrie R, Alexander N, et al. Diagnosing dying in the acute hospital setting-are we too late? Clin Med 2009;9:116-19.

38 Field D, Addington-Hall J. Extending specialist palliative care to all? Soc Sci Med 1999;48:1271-80.

39 Winzelberg GS, Hanson LC, Tulsky JA. Beyond autonomy: diversifying end-of-life decision-making approaches to serve patients and families. J Am Geriatr Soc 2005;53:1046-50.

40 Holdsworth L, Fisher S. A retrospective analysis of preferred and actual place of death for hospice patients. Int J Palliat Nurs 2010;16:424-30.

41 Tilden VP, Tolle SW, Drach LL, et al. Out-of-hospital death: advance care planning, decedent symptoms, and caregiver burden. J Am Geriatr Soc 2004;52:532-9.

42 Howell DA, Roman E, Cox H, et al. Destined to die in hospital? Systematic review and meta-analysis of place of death in haematological malignancy. BMC Palliat Care 2010;9:9.

43 Howat A, Veitch C, Cairns W. A retrospective review of place of death of palliative care patients in regional north Queensland. Palliat Med 2007;21:41-7.

44 O'Leary N, Tiernan E. Survey of specialist palliative care services for noncancer patients in Ireland and perceived barriers. Palliat Med 2008;22:77-83.

45 Tolle SW, Tilden VP, Rosenfeld AG, et al. Family reports of barriers to optimal care of the dying. Nurs Res 2000;49:310-17.

46 Murray CJL, Lopez AD. Alternative projections of mortality and disability by causes 1990-2020: global burden of disease. Lancet 1997;349:1498-504. 